\title{
MiR-99a Inhibits Cell Proliferation and Tumorigenesis through Targeting mTOR in Human Anaplastic Thyroid Cancer
}

\author{
Hou-Gang Huang ${ }^{1}$ Xi Luo ${ }^{1}$, Shuai $\mathrm{Wu}^{2}$, Bin Jian²*
}

\begin{abstract}
MicroRNAs (miRNAs) are emerging as critical regulators in carcinogenesis and tumor progression. Recently, miR-99a has been reported as a tumor suppressor gene in various human cancers, but its functions in the context of anaplastic thyroid cancer (ATC) remain unknown. In this study, we reported that miR-99a was commonly downregulated in ATC tissue specimens and cell lines with important functional consequences. Overexpression of miR-99a not only dramatically reduced ATC cell viability by inducing cell apoptosis and accumulation of cells at G1 phase, but also inhibited tumorigenicity in vivo. We then screened and identified a novel miR-99a target, mammalian target of rapamycin (mTOR), and it was further confirmed by luciferase assay. Up-regulation of miR-99a would markedly reduce the expression of mTOR and its downstream phosphorylated proteins ( $p$-4EBP1 and $p$-S6K1). Similar to restoring miR-99a expression, mTOR down-regulation suppressed cell viability and increased cell apoptosis, whereas restoration of mTOR expression significantly reversed the miR-99a antitumor activity and the inhibition of $\mathrm{mTOR} / p-4 \mathrm{E}-\mathrm{BP} 1 / p$-S6K1 signal pathway profile. In clinical specimens and cell lines, mTOR was commonly overexpressed and its protein levels were statistically inversely correlated with miR-99a expression. Taken together, our results demonstrated for the first time that miR-99a functions as a tumor suppressor and plays an important role in inhibiting the tumorigenesis through targeting the $\mathrm{mTOR} / p$ 4E-BP1/p-S6K1 pathway in ATC cells. Given these, miR-99a may serve as a novel prognostic/diagnostic and therapeutic target for treating ATC.
\end{abstract}

Keywords: MicroRNA-99a - mTOR - anaplastic thyroid cancer

Asian Pac J Cancer Prev, 16 (12), 4937-4944

\section{Introduction}

Anaplastic thyroid cancer (ATC) is one of the most aggressive types of malignant tumor with less than 8 months of mean survival after diagnosis (Ain, 1998). Although ATC represents less than $2 \%$ of all thyroid tumors, this neoplasm causes up to $14 \%$ 39\% of thyroid cancer-related deaths (Shaha, 2004). The current treatments for patients with ATC include surgery, radiotherapy and chemotherapy. Nevertheless, these therapies rarely succeed in improving the prognosis for patients with ATC (Wang et al., 2014; Zhang et al., 2014; Wang et al., 2015). Given this, there is an urgent need to develop novel strategies for the diagnosis, treatment and prognosis of ATC.

MicroRNAs (miRNAs) are a group of endogenous, 18 25 nucleotides non-coding RNA molecules that are highly conserved in eukaryotic organisms. These miRNAs can regulate gene expression post-transcriptionally through binding to the 3'-untranslated region (3'-UTR) of target genes to promote mRNA degradation or protein translation inhibition (Bartel, 2009). Thus, they operate highly complex regulatory networks and impact the expression of genes in many pathways that are associated with tumor initiation, development and progression (He et al., 2005b; Calin and Croce, 2006; Esquela-Kerscher and Slack, 2006; Valencia-Sanchez et al., 2006).Recent evidence has shown that about half of the human miRNAs are located in cancer-associated genomic regions that are frequently amplified, deleted, or rearranged in cancer, suggesting that some miRNAs may act as as tumor suppressor genes or oncogenes (Mutallip et al., 2011; Song et al., 2011; Fang et al., 2012). To date, several human miRNAs have been shown to be dysregulated in ATC, such as miR-221, miR-146, miR-200, and miR-17-92 cluster (He et al., 2005a; Takakura et al., 2008; Zhang et al., 2012), which contribute to the development and progression of ATC. These findings suggest the involvement of miRNAs in ATC tumorigenesis.

miR-99a is one of the members of the miR-99 family and has been reported to be downregulated in ovarian carcinoma, squamous cell carcinoma of tongue, squamous cell lung cancer, hepatocellular carcinoma, bladder cancer, prostate cancer and renal cell carcinoma (Nam et al., 2008; Wong et al., 2008; Catto et al., 2009; Gao et al., 2011; Li et al., 2011; Sun et al., 2011; Cui et al.,

${ }^{1}$ Department of Anaesthesiology, ${ }^{2}$ Department of Gastrointestinal Surgery, Yongchuan Hospital of Chongqing Medical University, Chongqing Medical University, Chongqing, China ¿Equal contributors*For correspondence: jianbin198028262@sina.com 
2012). These findings demonstrate miR-99a antitumor activity in different human cancers as a tumor suppressor. However, up to date, there has been no study reporting the role of miR-99a in ATC. Thus, our study investigated the biological functions and mechanisms of miR-99a as antitumor miRNA by repressing the activity of mTOR in ATC cells in vitro as well as in nude mouse xenografts. Finally, we explored the underlying mechanism of miR99a functions in ATC. Our study will provide a better understanding of ATC pathogenesis.

\section{Materials and Methods}

\section{Clinical ATC samples}

22 surgical specimens (both tumor and adjacent normal tissue) were obtained from patients in Yongchuan Hospital of Chongqing Medical University (Chongqing, China). The histological diagnosis was verified by an experienced pathologist. Tissue samples were immediately frozen in liquid nitrogen and stored at $-80^{\circ} \mathrm{C}$ until later used. No patient had received chemotherapy or radiation therapy treatment before surgery. The study was approved by the Institutional Review Boards of Yongchuan Hospital of Chongqing Medical University. The ethics committees approved this procedure. Informed consent for the experimental use of surgical specimens was obtained from all patients in written form according to the hospital's ethical guidelines.

\section{Cell lines and cell culture}

Normal human thyroid follicular epithelial cell line Nthy-ori 3-1 and human ATC cell lines SW1736, ARO and FRO were obtained from the State Key Laboratory of Molecular Oncology, Chinese Academy of Medical Sciences (Beijing, China). All cells were cultured in RPMI-1640 medium supplemented with 5\% FBS, $100 \mathrm{U} /$ $\mathrm{ml}$ penicillin and $100 \mathrm{mg} / \mathrm{ml}$ streptomycin in a humidified atmo $\neg$ sphere with $5 \% \mathrm{CO} 2$ at $37^{\circ} \mathrm{C}$.

\section{miRNA/siRNA/cDNA transfections}

miR-99a mimics, mTOR siRNA and negative control (NC) were chemically synthesized by GenePharma Biotechnology (Shanghai, China). ATC cells were plated in 6-well plates at $50 \%$ confluence the day before transfection. The next day, 50nM of mTOR siRNA or miR-99a mimics was transfected into ATC cells using Lipofectamine 2000 (Invitrogen, Carlsbad, USA) according to the manufacturer's protocol. Total RNAs or proteins were extracted at $72 \mathrm{~h}$ post-transfection and used for quantitative reverse transcription (qRT)- PCR or western blot analysis respectively.

The mTOR cDNA plasmid without 3'-UTR was purchased from Addgene (Cambridge, USA). For the mTOR cDNA and miR-99a mimics combination experiment, ATC cells were first transfected with miR-99a mimics $(50 \mathrm{nM}) .48 \mathrm{~h}$ later, these cells were co-transfected with mTOR cDNA plasmid ( $2 \mathrm{mg}$ ) and miR-99a mimics $(50 \mathrm{nM})$ for another $72 \mathrm{~h}$. Cells were used for following apoptosis assays or western blot analyses.

The lentivirus vector Lv-miR-99a which overexpress miR-99a and negative control LV-NC (non-targeting control sequence) were purchased from OriGene Technologies (Rockville, USA). Cells were transfected with LV-miR-99a (2 mg) or LV-NC (2 mg) using Lipofectamine 2000 according to manufacturer's instructions. For the in vivo tumorigenicity assay, we generated stable cell lines of ARO transfected by either LV-miR-99a or LV-NC, which were then named ARO-99a and ARO-NC, respectively.

\section{RNA extraction and $q R T-P C R$ analyses}

Total cellular RNA was extracted from ATC cells and tissues using TRIzol reagent (Invitrogen, Carlsbad, USA) according to the manufacturer's protocol. Expression of miR-99a was detected with the All-in-OneTM miRNA qRT-PCR Detection Kit (GeneCopoeia, Rockville, USA) and normalized to U6 snRNA using the 2- $(\Delta \Delta \mathrm{Ct})$ method. mTOR mRNA expression was quantified using One Step SYBR PrimeScript RT-PCR Kit II (Takara, Shiga, Japan) and normalized by GAPDH using the 2- $(\Delta \Delta \mathrm{Ct})$ method. The PCR primers were as follows: miR-99a, (forward) 5'-AAC CCG TAG ATC CGA TCT TGT G-3' and (reverse) 5'-TGG TGT CGT GGA GTC G-3'; U6, (forward) 5'-GCT TCG GCA GCA CAT ATA CTAAAA T-3' and (reverse) 5'-CGC TTC ACGAAT TTG CGT GTC AT-3'; mTOR, (forward) 5'-ACA GCC CAG GCC GCA TTG TC-3' and (reverse) 5'-TCC AGG GAC TCC GTC AGG CG-3'; GAPDH, (forward) 5'-CAATGA CCC CTT CAT TGA CC-3' and (reverse) 5'-GAC AAG CTT CCC GTT CTC AG-3'. All qRT-PCR amplifications were performed in triplicate.

\section{Western blot}

Cell extracts were prepared in ice-cold RIPA lysis buffer (Santa Cruz Biotechnology, Dallas, USA). After whole cell protein extracts were quantified by BCA protein assay, equivalent amounts of cell lysates were resolved by $10 \%$ SDS polyacrylamide gel electrophoresis and transferred onto PVDF membranes (Millipore, Billerico, USA), which was then blocked in $5 \%$ nonfat milk in PBST for 1 hour at $4^{\circ} \mathrm{C}$. The blots were then incubated with primary antibodies against mTOR, phosphorylated 4E-BP1, phosphorylated S6K1, 4E-BP1, S6K1 and GAPDH (Cell Signaling Technology, Danvers, USA). After incubation with horseradish peroxidaseconjugated secondary antibody (Santa Cruz, Dallas, USA), protein bands were visualized using enhanced chemiluminescence detection kit (Millipore, Billerico, USA). The intensity of the bands was analyzed using Image-Pro Plus software (version 6.0; Media Cybernetics, Rockville, USA). Each experiment was performed in triplicate.

\section{Luciferase reporter assay}

The wild-type 3'-UTR fragment of human mTOR mRNA containing the putative miR-99a binding site was amplified by PCR and cloned into the XbaI site of the pGL3-control vector (Promega, Madison, USA) which is the downstream of the luciferase reporter gene. The corresponding mutant constructs were created by mutating the seed regions of the miR-99a-binding sites (5'UAC GGG U-3' to 5'-AUG CCC A-3'). The nucleotide sequences of primers for mTOR 3'-UTR clone were: 
(forward) 5'-CGG GGT ACC AGA TGT GCC CAT CAC GTT TT-3' and (reverse) 5'-CCG GAA TTC TGG TGT CTA GAC ATG GCT ACA CTT-3', the mutagenesis primers were: (forward) 5'-CCA TAA CTT TAG AAA GCT ACA CTT TGA CTT AAC TCA C-3' and (reverse) 5'-GTG AGT TAA GTC AAA GTG TAG CTT TCT AAA GTT ATG G-3'. For the luciferase reporter assay, the 293T cell line was co-transfected with luciferase reporter vectors and miR-99a mimics using Lipofectamine 2000. A 1-ng pRL-TK Renilla Luciferase construct was used for normalization. After $48 \mathrm{~h}$, the luciferase activity was analyzed by the Dual-Luciferase Reporter Assay System according to the manufacture's protocols (Promega, Madison, USA).

\section{Cell viability assay}

Cell viability capacity was assessed by detection of formazan production from the CellTiter 96 AQueous One Solution Cell Proliferation Assay kit (Promega, Madison, USA). Briefly, cells were plated in 96-well plates at a density of $1 \times 10^{4}$ cells/well and then transfected with miR-99a mimics, mTOR siRNA, or negative control using Lipofectamine 2000 according to manufacturer's instructions. The colorimetric MTT assay was performed to measure the absorbance rate of cells and the samples were detected at $0,24 \mathrm{~h}, 48 \mathrm{~h}, 72 \mathrm{~h}$ and $96 \mathrm{~h}$ after gene transfection. This assay was performed in triplicate and repeated thrice.

Flow cytometry analysis of apoptosis and cell cycle

After transfected with miR-99a mimics, mTOR siRNA, or negative control for $72 \mathrm{~h}$, the cells were incubated in serum-free medium for $24 \mathrm{~h}$ for cell synchronization. For apoptosis analysis, cultured cells were harvested by trypsinization and washed twice by $4^{\circ} \mathrm{C}$ precooling PBS. Cells $\left(1 \times 10^{6}\right.$ cells $\left./ \mathrm{ml}\right)$ from each sample were processed with the Annexin V-FITC/PI apoptosis detection kit (BD Biosciences, San Jose, USA) according to the manufacturer's instructions. For cell cycle analysis, the cells were typsinized, washed twice with PBS, and then fixed with $75 \%$ ethanol on ice for $2 \mathrm{~h}$. The fixed cells were centrifuged and washed twice in cold PBS; treated with $25 \mu \mathrm{g} / \mathrm{ml} \mathrm{RNase} \mathrm{A} \mathrm{at} 37^{\circ} \mathrm{C}$ for $30 \mathrm{~min}$ and stained with propidiumiodide (PI) $(50 \mu \mathrm{g} / \mathrm{ml}$, Sigma) solution for $30 \mathrm{~min}$ in the dark. The fluorescence intensity of individual cells was measured by a flow cytometer (Becton Dickinson, USA). The experiments were performed in triplicate and repeated thrice.

\section{Nude mouse tumor xenograft model}

To assess the inhibitory effect of miR-99a in ATC cells, we performed the in vivo tumor xenograft assay using 30 female, 4 to 5 week-old BALB/c nude mice, which were randomly divided into three treatment groups with 10 mice in each group, i.e. ARO cells with LV-miR-99a, LV-NC, and MOCK groups. The mice received subcutaneous injection of $3 \times 10^{6}$ cells in the left flank respectively in 100 $\mathrm{ml}$ of serum-free medium. Tumor size was measured for length and width every 5 days for 30 days, and volume was calculated using the formula: volume $=\left(\right.$ length $\times$ width $\left.{ }^{2}\right) / 2$. All experimental procedures involving the use of animals were in accordance with the Guide for the Care and Use of Laboratory Animals and were approved by the ethics committee of the Yongchuan Hospital of Chongqing Medical University.

\section{Statistical analysis}

Each experiment was repeated at least three times. Quantitative data were summarized as mean \pm SD. The differences and correlations between two groups were assessed by the Mann-Whitney U test or Pearson's correlation test. Multiple group comparison was analyzed by using ANOVA with a post-test for subsequent individual group comparisons. All statistical analyses were performed using SPSS 13.0 (SPSS, Chicago, USA). Data were considered to be statistically significant when $p<0.05$.

\section{Results}

The miR-99a expression is down-regulated in both human ATC tissues and cell lines

To investigate the biological role of miR-99a in ATC, we firstly performed real-time qRT-PCR using ATC tissue from patients and found that miR-99a expression was reduced in all 22 cases of human ATC tissues compared to the normal tissue counterparts (Figure 1A). Moreover, we also detected miR-99a expression in three ATC cell lines (i.e.SW1736, ARO and FRO) and a normal human thyroid follicular epithelial cell line Nthy-ori 3-1. As shown in Figure 1B, miR-99a expression was significantly reduced in ATC cancer cell lines compared to Nthy-ori 3-1 cells. These data suggest that the downregulation of miR-99a is clearly involved in ATC carcinogenesis.

Overexpression of miR-99a inhibits ATC cancer cell viability and induces apoptosis

The reduced expression of miR-99a in ATC prompted us to identify whether miR-99a functions as a tumor suppressor. To investigate the role of miR-99a, we established a miR-99a over-expression model by transfecting with miR-99a mimics into ARO cells, and

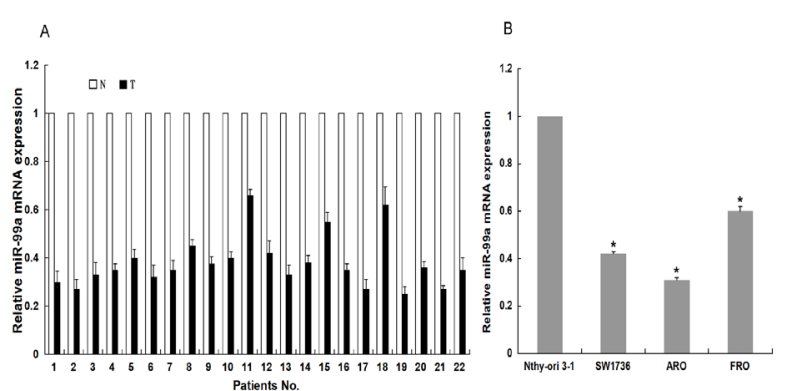

Figure 1. Differential Expression of miR-99a in Human ATC Tissues and Cell Lines. (A) qRT-PCR analysis of miR-99a expression in 22 human ATC tissues vs. the distant non-tumor tissues. (B) qRT-PCR analysis of miR-99a expression in three human ATC cell lines and a normal human thyroid follicular epithelial cell line. The level of miR-99a expression was normalized to U6 and compared to the normal cells. Data were normalized to $\mathrm{U} 6$ snRNA and represent the mean $\pm \mathrm{SD}$ of three independent duplicate experiments. ${ }^{*} \mathrm{P}<0.05$ significant differences from Nthy-ori 3-1 


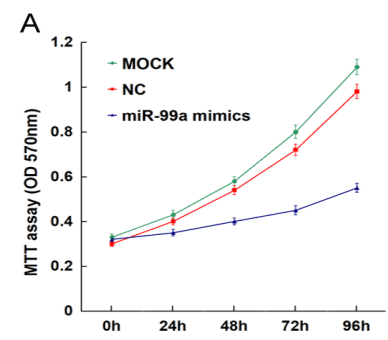

B
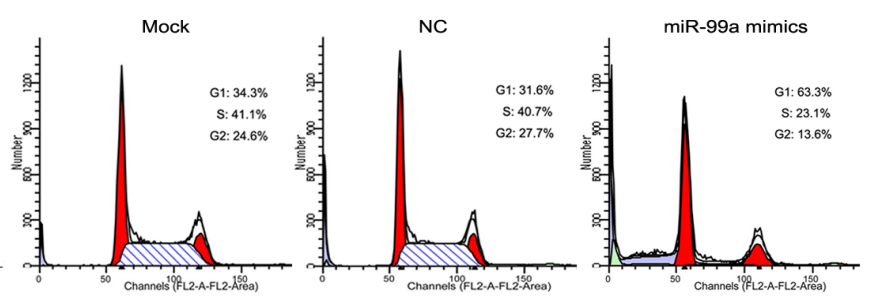

$\mathrm{E}$
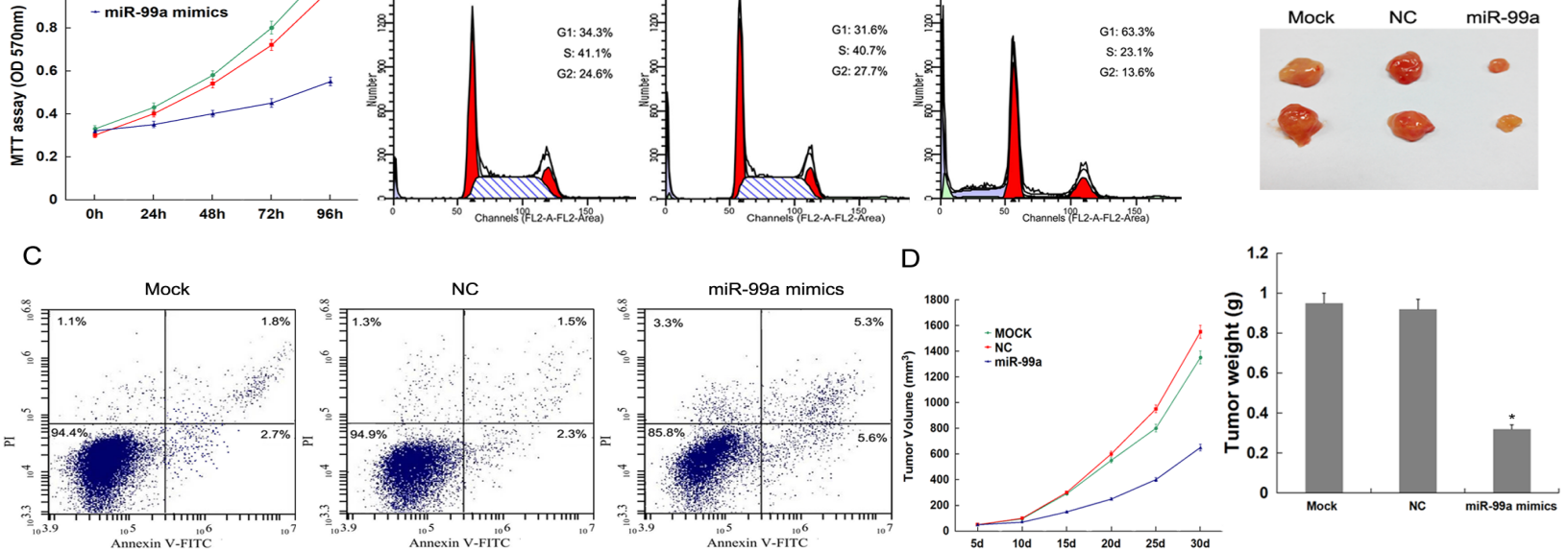

Figure 2. The Inhibitory Effect of miR-99a Mimics on ATC Cells in vitro and in vivo. (A) MTT assay of cell viability. The cell viability curve was constructed consecutively for up to 96h after transfection of ARO cells with Lipofectamine 2000 only (MOCK), scrambled negative control (NC) and miR-99a mimics. (B) Flow cytometry analysis of cell cycle. At $72 \mathrm{~h}$ post-transfection, ARO cell lines treated with miR-99a mimics showed a remarkable increased G1 phase population compared with MOCK and NC groups. (C) Flow cytometry analysis of apoptosis. After 72h treatment, transfection of miR-99a mimics into ARO cell lines resulted in a significant increase percentage of apoptotic cells compared with the control. (D) Tumor size observation in nude mice after ATC cell inoculation. The average size of the tumors was measured every 5 days and shown in the curves. (E) Tumor sizes of representative nude mice. The tumor weight was measured at the final time point (30-day). All experiments were carried 3 times independently. $* P<0.05$ compared to the control cells
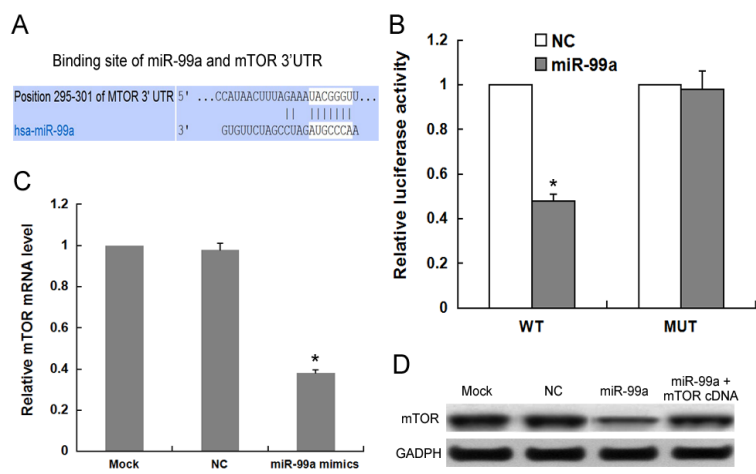

Figure 3. mTOR is a Direct Target of miR-99a in ATC

Cell Line. (A) Targetscan prediction of miR-99a targeting of mTOR at the mTOR mRNA 3'-UTR. (B) The relative luciferase activity of the reporter containing 3'-UTR of mTOR was suppressed by miR-99a, but not in the mutated construct. qRT-PCR (C) and western blot (D) analyses were performed to examine the effects of miR-99a on mTOR expression in ARO cells. The expression of mTOR was suppressed after miR-99a overexpression. The suppression was abolished by transfection of the cells with mTOR cDNA without 3'-UTR. All experiments were carried 3 times independently. $* \mathrm{P}<0.05$ compared to the control cells

cells transfected with Lipofectamine 2000 only (MOCK) and negative control (NC) were used as controls. Using cell proliferation assay, we observed that miR-99a overexpression in ARO cells led to a notable reduction of cell viability compared to control cells (Figure 2A, $p<0.05)$. Cell cycle analyses indicated that transfection of miR-99a mimics resulted in a remarkable accumulation of cell cycle at G1 phase, which suggests cells undergoing apoptosis (Telford et al., 1992) (Figure 2B, $p<0.05$ ). Similarly, the effect of miR-99a mimics on inducing cell apoptosis was significantly increased compared to the control groups (Figure $2 \mathrm{C}, p<0.05$ ). Together, these results indicate that miR-99a overexpression clearly inhibits the proliferation of human ATC cells in vitro.

miR-99a overexpression suppresses tumor growth in vivo

To further confirm the in vitro results above, we performed a nude mouse xenograft assay by injecting ARO cells stably transfected with LV-miR-99a into nude mice. We detected that tumor growth in the LV-miR-99a group was significantly reduced than that of the control groups (Figure 2D). The average tumor weight of the LV-NC group was 2.77 fold higher than that of the LV-miR-99a group after 30 days post-inoculation (Figure 2E).

\section{MTOR is a direct target of miR-99a in ATC cancer cells}

To explore the mechanisms by which miR-99a regulates the tumorigenicity of ATC, we performed a bioinformatic search (Targetscan, Pictar and MICROCOSM) for putative targets of miR-99a and found that mTOR was one of the predicted miR-99a targets whose 3'-UTR contained putative miR-99a target sites (Figure 3A). This finding above was consistent with previous studies in different human cancers (Li et al., 2011; Sun et al., 2011; Cui et al., 2012; Sun et al., 2013). To confirm that mTOR is a direct target of miR-99a, we cloned the 3'-UTR of mTOR into a reporter plasmid downstream from luciferase and performed reporter assays. The result demonstrated that the relative luciferase activity of the reporter which contained wild-type 3'-UTR of mTOR was notably decreased in miR-99a group compared to NC group. In contrast, the relative luciferase activity of the mutant mTOR 3'-UTR reporter showed no significant difference with the control group and failed to respond to miR-99a (Figure 3B). To further determine whether miR-99a had a functional role in down-regulation of mTOR expression in human ATC 


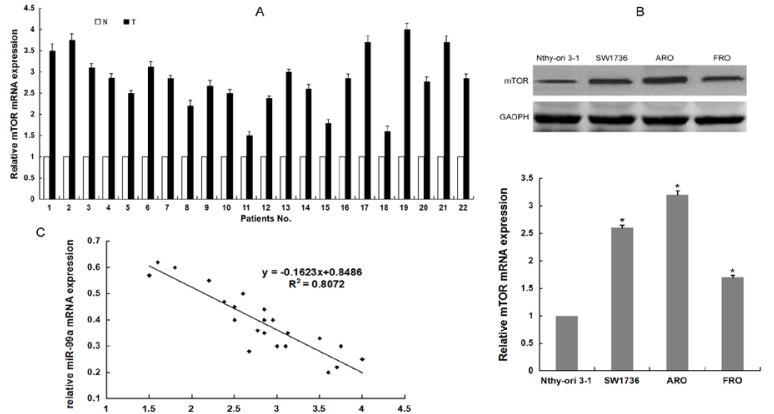

Figure 4. Statistical Correlation between the Expression Levels of miR-99a and mTOR in ATC Tissues and Cell Lines. (A) mTOR mRNA level was analyzed by qRT-PCR in 22 pairs of ATC tissues. (B) mTOR protein and mRNA were analyzed in three different ATC cell lines and a normal human thyroid follicular epithelial cell line. $* \mathrm{P}<0.05$ significant differences from Nthy-ori 3-1. (C) The expression of miR-99a and MTOR is negatively correlated in clinical ATC tissues. The inverse correlation in ATC tissues was analyzed by Pearson's correlation method

cells, the mTOR expression was determined at mRNA and protein levels by qRT-PCR and immunoblot. In ARO cells transfected by miR-99a mimics, mTOR was significantly suppressed at the levels of both mRNA ( 0.38 fold) and protein ( 0.41 fold) compared to control groups (Figure $3 \mathrm{C})$. However, transfection with mTOR cDNA plasmid that lacks 3'-UTR could dramatically overcome the repressive effect of miR-99a mimics (Figure 3D). Taken together, these findings identified $\mathrm{mTOR}$ as a direct miR99a target and suggested that miR-99a overexpression suppresses the expression of mTOR in ARO cells.

MiR-99a negatively correlates with mTOR expression in both human ATC tissues and cell lines

To further confirm that miR-99a inhibits mTOR expression in ATC cancer, we analyzed the mRNA levels of mTOR from 22 pairs of ATC and normal tissue specimens by qRT-PCR. Compared to the non-tumor counterparts, the expression of mTOR mRNA was significantly increased in tumor tissues from 22 different patients (Figure 4A). Similar results was also demonstrated in ATC cells that mTOR expression in these three ATC cell lines were markedly higher than that in normal thyroid follicular epithelial cell line, and was associated with reduced miR99a expression (Figure 4B). Correlation analyses among these ATC samples was then performed, which indicated that there was a statistically significant inverse correlation between $\mathrm{mTOR}$ and miR-99a expression (Figure 4C, R2= 0.8072, Pearson $\mathrm{X}^{2}$ test).

\section{MiR-99a-mediated mTOR inhibition is involved in tumor suppression of ATC cells}

To further elucidate whether the antitumor activity of miR-99a was mediated by down-regulation of mTOR in ATC cells, we performed gain-of-function and lossof-function studies. First, we inhibited endogenous mTOR expression to assess whether it can mimic the biological effect of miR-99a. Indeed, knockdown of mTOR expression by mTOR-siRNA caused a significant reduction of cell viability and a notable increase in cell apoptosis compared to the control group, in a manner similar to those transfected with miR-99a mimics (Figure $5 \mathrm{~A})$. Next, we evaluated whether the ectopic expression of mTOR could reverse the suppressive effect of miR99a on ATC cells. ARO cells were co-transfected with miR-99a mimics and mTOR cDNA plasmid that lacks the 3'-UTR, and the results indicated that the growth inhibition and apoptosis acceleration of ATC cells by miR-99a mimics were rescued by restoration of mTOR expression (Figure 5B).

miR-99a regulates the expression of key components of the MTOR signaling pathway in ATC cells

We then sought to determine whether the mTORmediated downstream signal pathway was also impacted
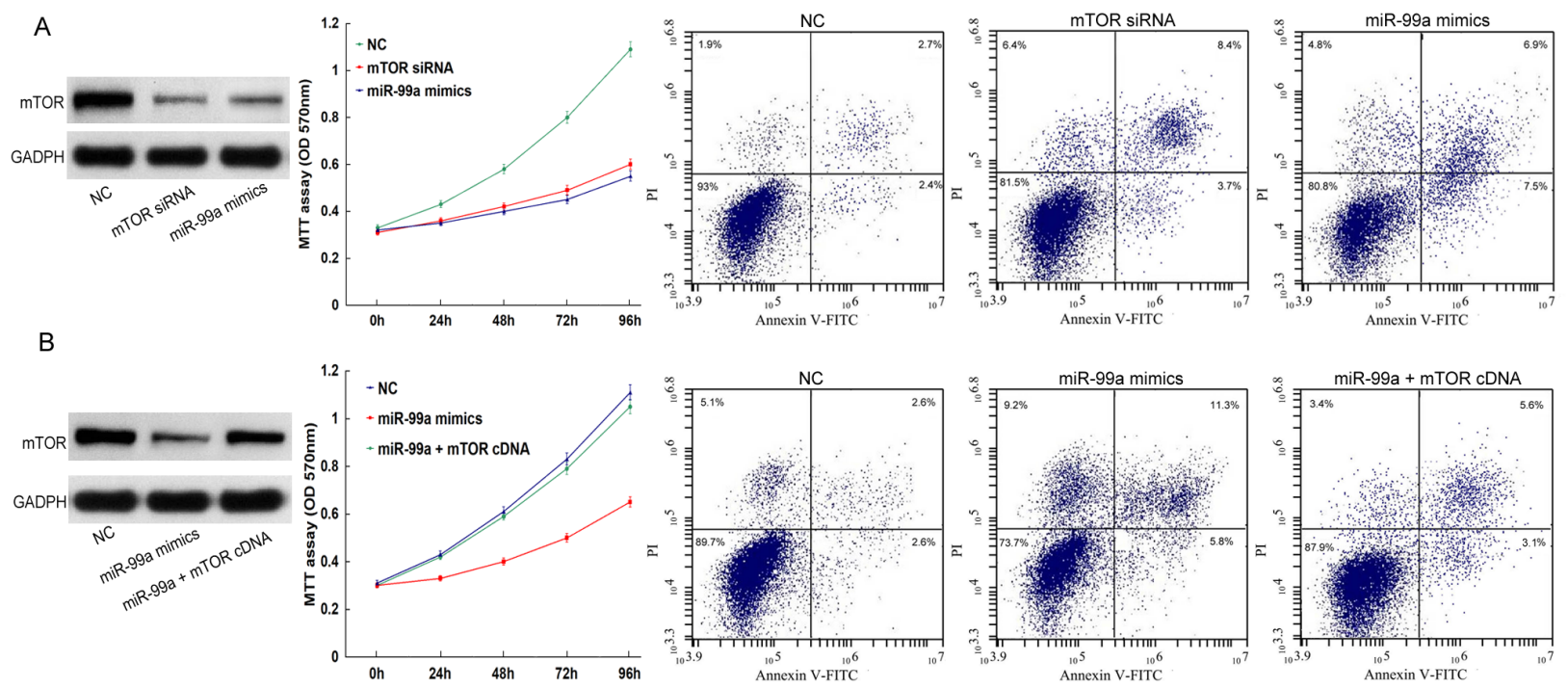

Figure 5. mTOR is Involved in miR-99a-mediated Antitumor Activity in ATC Cells. (A) ARO cells were transfected with NC, mTOR siRNA and miR-99a mimics respectively. Cell viability assay and cell apoptosis were measured at $72 \mathrm{~h}$ posttransfection. (B) ARO cells were transfected with NC, miR-99a mimics and mTOR cDNA plasmid without the 3'-UTR respectively. Cell viability assay and cell apoptosis were measured at $72 \mathrm{~h}$ post-transfection 


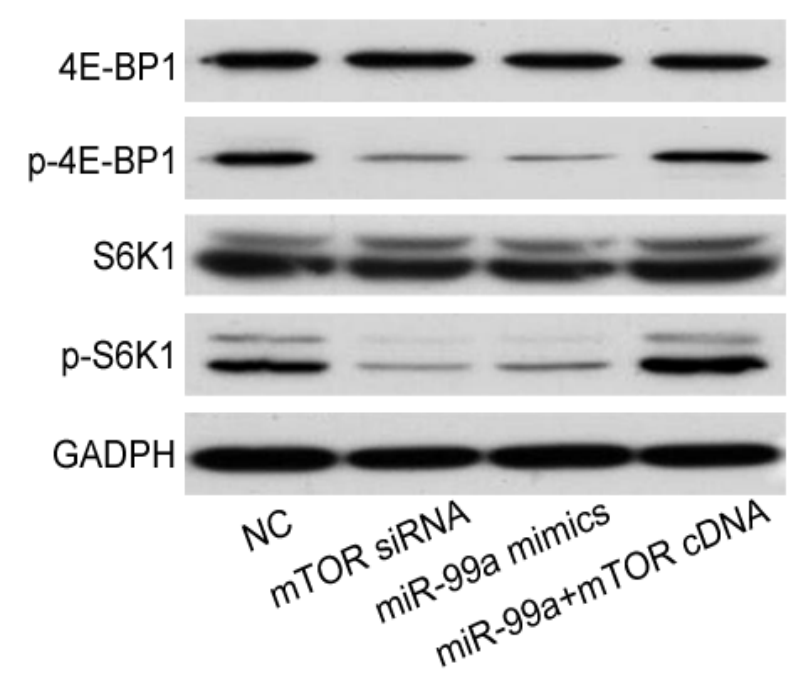

Figure 6. miR-99a Regulated Expression of mTOR Downstream Signaling Pathway Genes $p$-4E-BP1 and $p$-S6K1. The overexpression of miR-99a significantly suppressed the mTOR/p-4E-BP1/p-S6K 1 signal pathway examined by western blot. The inhibition of $\mathrm{mTOR} / p-4 \mathrm{E}-\mathrm{BP} 1 / p$ S6K1 was reversed by re-expression of mTOR cDNA in miR99a group. All experiments were carried 3 times independently. * $\mathrm{P}<0.05$ compared to control group

by miR-99a. To achieve this goal, downstream substrates of mTOR pathway were investigated after transfection of miR-99a mimics in ARO cells, and we found that the endogenous expression of both $p$-4EBP1 and $p$-S6K1, major components of the mTOR pathway, were markedly decreased at protein levels compared to the control cells (Figure 6).Interestingly, however, total 4E-BP1 and S6K1 expression remained the same. Furthermore, re-expression of mTOR in ARO cells could significantly overcome the inhibitory effects of miR-99a on $\mathrm{mTOR} / p-4 \mathrm{E}-\mathrm{BP} 1 / p$ S6K1 signal pathway (Figure 6). Taken together, these data suggests an important role of mTOR as a mediator of the biological effects of miR-99a.

\section{Discussion}

In the last decade, miRNAs have emerged as critical regulators in cancer-related processes (Hummel et al., 2010; Shibuya et al., 2010), and tumor-targeting therapy using miRNAs is becoming a novel diagnostic and therapeutic tool (Iorio and Croce, 2009). Previous studies have reported that miR-99a participated in tumorigenesis of several tumor types, including hepatocellular carcinoma, prostate cancer, renal cell carcinoma, and lung cancer (Li et al., 2011; Oneyama et al., 2011; Sun et al., 2011; Cui et al., 2012). However, the effect of miR-99a on anaplastic thyroid cancer (ATC) has not been reported so far. In this study, we demonstrate for the first time that miR-99a is implicated in the carcinogenesis of ATC. Compared with human normal thyroid follicular epithelial cell line Nthy-ori 3-1, the expression of miR-99a was significantly downregulated in ATC cell lines SW1736, ARO and FRO. The detection of miR-99a in 22 ATC tissue samples also pointed to a dramatic attenuation of miR99a expression, which was consistent with the results in cell lines. These results indicate that miR-99a may serve as a potential predictor for prognosis of ATC patients. A limitation to our study was the relatively small number of clinical samples at our disposal. Further studies with more clinical samples are warranted.

The reduction of miR-99a expression in ATC prompted us to identify whether miR-99a functions as a tumor suppressor. It has been reported that overexpression of miR-99a by transient gene transfection inhibited esophageal cancer cell proliferation and induced apoptosis (Sun et al., 2013). MiR-99a was also found to induce cell cycle arrest at G1 phase and suppress tumorigenicity in renal cell carcinoma (Cui et al., 2012). In addition, Expression of miR-99a inhibits the growth of prostate cancer cells and reduces the expression of prostate-specific antigen (PSA) (Sun et al., 2011). In our study, we found that ectopic expression of miR-99a greatly decreased ATC cell viability, induced G1-phase cell cycle arrest and apoptosis in vitro. Moreover, in vivo tumor growth was suppressed with exogenous miR-99a expression in ATC xenograft model. These findings were consistent with former reports in different cancers and suggested that miR-99a plays a tumor suppressive role and may be a therapeutic intervention in ATC.

On the basis of bioinformatics analyses and sequential experimental demonstration, mTOR was identified as a direct target of miR-99a in ATC. MTOR is a phosphatidylinositol kinase-related kinase known to mediate cellular responses to growth factors and regulate cell proliferation, metabolism, and angiogenesis (Petroulakis et al., 2006). Previous studies have reported that miR-99a can inhibit mTOR expression by directly targeting its 3'-UTR in numerous human cancers including prostate cancer, childhood adrenocortical tumor, renal cell carcinoma and gastrointestinal stromal tumor (Doghman et al., 2010; Sun et al., 2011; Cui et al., 2012; Li et al., 2013). Our present experimental results confirm that mTOR, as well as other human cancers just mentioned, are functional targets of miR-99a in ATC cells. There are several lines of evidence to support this. Firstly, miR-99a restoration significantly down-regulated mTOR expression at both protein and transcript levels by directly targeting the 3'-UTR of mTOR mRNA, which was confirmed using luciferasereporter-gene assays. This effect was largely eliminated when the sites in mTOR 3'-UTR targeted by miR-99a were mutated. Our clinical data also showed a statistically significant inverse correlation between expression of mTOR and miR-99a in ATC tissues. Moreover, the repression of mTOR expression by miR99a was rescued by transfection of mTOR cDNA plasmid that lacks the 3'-UTR. These findings strongly suggested that miR-99a directly inhibits mTOR expression in ATC via binding to mTOR 3'-UTR.

MTOR is a protein kinase in the PI3K/Akt signaling pathway and mTOR signaling pathway is a key signaltransduction system that links multiple receptors and oncogenic molecules to diverse cellular functions (Guertin and Sabatini, 2007). MTOR protein can phosphorylate and activate its key downstream effectors 4E-BP1 and S6K1, in control of protein translation (such as CDK, cyclin D1 and cyclin E) and regulate cell proliferation and cell 
cycle (Hara et al., 2002; Kim et al., 2002). Abnormality of mTOR signaling appears to directly interfere with the normal cell growth and apoptosis in many cancer types such as prostate cancer, lung cancer, acute myelogenous leukemia and hepatocellular carcinoma (Kremer et al., 2006; Martelli et al., 2009; Cui et al., 2012; Ekman et al., 2012; Grabinski et al., 2012). To further elucidate mechanisms underlying the tumor suppressive effect of miR-99a in ATC, we knockdowned mTOR in ARO cells using mTOR siRNA and found that ATC cell viability was suppressed and apoptosis was induced, similar to that of tumor cells transfected with miR-99a mimics. Furthermore, the inhibition of cell viability and the acceleration of apoptosis by miR-99a mimics were rescued by restoration of $\mathrm{mTOR}$. These results indicated that mTOR was required for the miR-99a-dependent cell viability and apoptosis effect in ATC cells.

In mammals, S6K1 and 4E-BP1 are two best characterized downstream targets of mTOR (Bjornsti and Houghton, 2004). Activation of S6K1 protein enhances the translation of cellular mRNAs with a 5'-terminal oligopyrimidine tract and such mRNAs exclusively encode for components of the translation apparatus and control cell growth (Radimerski et al., 2002; Liu et al., 2006). $4 \mathrm{E}-\mathrm{BP} 1$ primarily binds tightly to the cap-binding protein eIF4E and represses cap-dependent mRNA translation, functioning as a suppressor of eIF4E (Mothe-Satney et al., 2000). The phosphorylation of 4E-BP1 at multiple site releases eIF4E to restore cap-dependent mRNA translation (such as cyclin D1 and c-Myc), which also promote cell growth (Gera et al., 2004; Liu et al., 2006). Inhibition of mTOR expression decreases expression of S6K1 and 4E$\mathrm{BP} 1$, and subsequently leads to the reduction of mRNAs translation for negative regulators of cell cycle progression and cell proliferation, such as cyclin D1, c-Myc, Bcl-2, BclxL and eIF4B (Fingar et al., 2004; Jastrzebski et al., 2007; Soni et al., 2008). On the contrary, overexpression of $4 \mathrm{E}-\mathrm{BP} 1$ or S6K1 leads to aggressive phenotype of various cancers, such as breast cancer, bladder cancer and rhabdomyosarcoma (Heinonen et al., 2008; Nawroth et al., 2011; Zhou and Huang, 2011; Tan et al., 2014) In our current study, we found that phosphorylation of S6K1 and 4E-BP1 was significantly suppressed after inhibition of mTOR expression by miR-99a mimics in ATC cell lines, concurrent with a reduction of cell viability and induction of apoptosis, while re-expression of mTOR could completely overcome the inhibitory effect of miR-99a on expression of $\mathrm{mTOR} / p-4 \mathrm{E}-\mathrm{BP} 1 / p-\mathrm{S} 6 \mathrm{~K} 1$ signal pathway genes. Thus, our study provides a strong support of miR-99-targeted mTOR/p-4E-BP1/p-S6K1 signaling pathway in ATC cells. In light of the discussion above concerning the inhibitory role of miRNA-99a with respect to mTOR-related signal pathway, it is implied that miRNA-99a might offer a potential target for prognostic/ diagnostic and therapeutic interventions in ATC. However, the mechanisms underlying miR-99a implicated in the carcinogenesis of ATC is very complicated, and further extensive analysis will be necessary to elucidate the precise mechanisms of miR-99a implicated in the carcinogenesis of ATC.

In conclusion, Our findings demonstrated for the first time that miR-99a was frequently downregulated in ATC cell lines and clinical samples. Upregulation of miR-99a reduced tumor cell tumorigenicity in vitro and in vivo through targeting mTOR. MTOR plays a critical role in mediating miR-99a dependent biological functions in ATC. Restoration of miR-99a might therefore lead to the design of novel therapeutic tools with which to treat ATC patients. Additional studies are warranted to examine the mechanism of actions of these proteins in the tumor process, as well as possible crosstalk with other signaling pathways.

\section{References}

Ain KB (1998). Anaplastic thyroid carcinoma: behavior, biology, and therapeutic approaches. Thyroid, 8, 715-26.

Bartel DP (2009). MicroRNAs: target recognition and regulatory functions. Cell, 136, 215-33.

Bjornsti MA, Houghton PJ (2004). The TOR pathway: a target for cancer therapy. Nat Rev Cancer, 4, 335-48.

Calin GA, Croce CM (2006). MicroRNA signatures in human cancers. Nat Rev Cancer, 6, 857-66.

Catto JW, Miah S, Owen HC, et al (2009). Distinct microRNA alterations characterize high- and low-grade bladder cancer. Cancer Res, 69, 8472-81.

Cui L, Zhou H, Zhao H, et al (2012). MicroRNA-99a induces G1-phase cell cycle arrest and suppresses tumorigenicity in renal cell carcinoma. BMC Cancer, 12, 546.

Doghman M, El Wakil A, Cardinaud B, et al (2010). Regulation of insulin-like growth factor-mammalian target of rapamycin signaling by microRNA in childhood adrenocortical tumors. Cancer Res, 70, 4666-75.

Ekman S, Wynes MW, Hirsch FR (2012). The mTOR pathway in lung cancer and implications for therapy and biomarker analysis. J Thorac Oncol, 7, 947-53.

Esquela-Kerscher A, Slack FJ (2006). Oncomirs - microRNAs with a role in cancer. Nat Rev Cancer, 6, 259-69.

Fang Y, Xue JL, Shen Q, et al (2012). MicroRNA-7 inhibits tumor growth and metastasis by targeting the phosphoinositide 3-kinase/Akt pathway in hepatocellular carcinoma. Hepatology, 55, 1852-62.

Fingar DC, Richardson CJ, Tee AR, et al (2004). mTOR controls cell cycle progression through its cell growth effectors S6K1 and 4E-BP1/eukaryotic translation initiation factor 4E. Mol Cell Biol, 24, 200-16.

Gao W, Shen H, Liu L, et al (2011). MiR-21 overexpression in human primary squamous cell lung carcinoma is associated with poor patient prognosis. J Cancer Res Clin Oncol, 137, 557-66.

Gera JF, Mellinghoff IK, Shi Y, et al (2004). AKT activity determines sensitivity to mammalian target of rapamycin (mTOR) inhibitors by regulating cyclin D1 and c-myc expression. J Biol Chem, 279, 2737-46.

Grabinski N, Ewald F, Hofmann BT, et al (2012). Combined targeting of AKT and mTOR synergistically inhibits proliferation of hepatocellular carcinoma cells. Mol Cancer, 11, 85 .

Guertin DA, Sabatini DM (2007). Defining the role of mTOR in cancer. Cancer Cell, 12, 9-22.

Hara K, Maruki Y, Long X, et al (2002). Raptor, a binding partner of target of rapamycin (TOR), mediates TOR action. Cell, 110, 177-89.

He H, Jazdzewski K, Li W, et al (2005a). The role of microRNA genes in papillary thyroid carcinoma. Proc Natl Acad Sci U $S$ A, 102, 19075-80.

He L, Thomson JM, Hemann MT, et al (2005b). A microRNA 
Hou-Gang Huang et al

polycistron as a potential human oncogene. Nature, $\mathbf{4 3 5}$, 828-33.

Heinonen H, Nieminen A, Saarela M, et al (2008). Deciphering downstream gene targets of $\mathrm{PI} 3 \mathrm{~K} / \mathrm{mTOR} / \mathrm{p} 70 \mathrm{~S} 6 \mathrm{~K}$ pathway in breast cancer. BMC Genomics, 9, 348 .

Hummel R, Hussey DJ, Haier J (2010). MicroRNAs: predictors and modifiers of chemo- and radiotherapy in different tumour types. Eur J Cancer, 46, 298-311.

Iorio MV, Croce CM (2009). MicroRNAs in cancer: small molecules with a huge impact. J Clin Oncol, 27, 5848-56.

Jastrzebski K, Hannan KM, Tchoubrieva EB, et al (2007). Coordinate regulation of ribosome biogenesis and function by the ribosomal protein S6 kinase, a key mediator of mTOR function. Growth Factors, 25, 209-26.

Kim DH, Sarbassov DD, Ali SM, et al (2002). mTOR interacts with raptor to form a nutrient-sensitive complex that signals to the cell growth machinery. Cell, 110, 163-75.

Kremer CL, Klein RR, Mendelson J, et al (2006). Expression of mTOR signaling pathway markers in prostate cancer progression. Prostate, 66, 1203-12.

Li D, Liu X, Lin L, et al (2011). MicroRNA-99a inhibits hepatocellular carcinoma growth and correlates with prognosis of patients with hepatocellular carcinoma. J Biol Chem, 286, 36677-85.

Li JC, Zhu HY, Chen TX, et al (2013). Roles of mTOR and $p$-mTOR in gastrointestinal stromal tumors. Asian Pac J Cancer Prev, 14, 5925-8.

Liu L, Li F, Cardelli JA, et al (2006). Rapamycin inhibits cell motility by suppression of mTOR-mediated S6K1 and 4EBP1 pathways. Oncogene, 25, 7029-40.

Martelli AM, Evangelisti C, Chiarini F, et al (2009). Targeting the $\mathrm{PI} 3 \mathrm{~K} / \mathrm{AKT} / \mathrm{mTOR}$ signaling network in acute myelogenous leukemia. Expert Opin Investig Drugs, 18, 1333-49.

Mothe-Satney I, Yang D, Fadden P, et al (2000). Multiple mechanisms control phosphorylation of PHAS-I in five (S/T)P sites that govern translational repression. Mol Cell Biol, 20, 3558-67.

Mutallip M, Nohata N, Hanazawa T, et al (2011). Glutathione S-transferase P1 (GSTP1) suppresses cell apoptosis and its regulation by miR-133alpha in head and neck squamous cell carcinoma (HNSCC). Int J Mol Med, 27, 345-52.

Nam EJ, Yoon H, Kim SW, et al (2008). MicroRNA expression profiles in serous ovarian carcinoma. Clin Cancer Res, 14, 2690-5.

Nawroth R, Stellwagen F, Schulz WA, et al (2011). S6K1 and 4E$\mathrm{BP} 1$ are independent regulated and control cellular growth in bladder cancer. PLOS ONE, 6, 27509.

Oneyama C, Ikeda J, Okuzaki D, et al (2011). MicroRNAmediated downregulation of mTOR/FGFR3 controls tumor growth induced by Src-related oncogenic pathways. Oncogene, 30, 3489-501.

Petroulakis E, Mamane Y, Le Bacquer O, et al (2006). mTOR signaling: implications for cancer and anticancer therapy. Br J Cancer, 94, 195-9.

Radimerski T, Montagne J, Rintelen F, et al (2002). dS6Kregulated cell growth is $\mathrm{dPKB} / \mathrm{dPI}$ (3)K-independent, but requires dPDK1. Nat Cell Biol, 4, 251-5.

Shaha AR (2004). Implications of prognostic factors and risk groups in the management of differentiated thyroid cancer. Laryngoscope, 114, 393-402.

Shibuya H, Iinuma H, Shimada R, et al (2010). Clinicopathological and prognostic value of microRNA-21 and microRNA-155 in colorectal cancer. Oncology, 79, 313-20.

Song YX, Yue ZY, Wang ZN, et al (2011). MicroRNA-148b is frequently down-regulated in gastric cancer and acts as a tumor suppressor by inhibiting cell proliferation. $\mathrm{Mol}$ Cancer, 10, 1.
Soni A, Akcakanat A, Singh G, et al (2008). eIF4E knockdown decreases breast cancer cell growth without activating Akt signaling. Mol Cancer Ther, 7, 1782-8.

Sun D, Lee YS, Malhotra A, et al (2011). miR-99 family of MicroRNAs suppresses the expression of prostate-specific antigen and prostate cancer cell proliferation. Cancer Res, 71, 1313-24.

Sun J, Chen Z, Tan X, et al (2013). MicroRNA-99a/100 promotes apoptosis by targeting mTOR in human esophageal squamous cell carcinoma. Med Oncol, 30, 411.

Takakura S, Mitsutake N, Nakashima M, et al (2008). Oncogenic role of miR-17-92 cluster in anaplastic thyroid cancer cells. Cancer Sci, 99, 1147-54.

Tan HK, Moad AI, Tan ML (2014). The mTOR signalling pathway in cancer and the potential mTOR inhibitory activities of natural phytochemicals. Asian Pac J Cancer Prev, 15, 6463-75.

Telford WG, King LE, Fraker PJ (1992). Comparative evaluation of several DNA binding dyes in the detection of apoptosisassociated chromatin degradation by flow cytometry. Cytometry, 13, 137-43.

Valencia-Sanchez MA, Liu J, Hannon GJ, et al (2006). Control of translation and mRNA degradation by miRNAs and siRNAs. Genes Dev, 20, 515-24.

Wang JN, Xu LH, Zeng WG, et al (2015). Treatment of human thyroid carcinoma cells with the g47delta oncolytic herpes simplex virus. Asian Pac J Cancer Prev, 16, 1241-5.

Wang JT, Huang R, Kuang AR (2014). Comparison of presentation and clinical outcome between children and young adults with differentiated thyroid cancer. Asian Pac J Cancer Prev, 15, 7271-5.

Wong TS, Liu XB, Wong BY, et al (2008). Mature miR-184 as potential oncogenic microRNA of Squamous Cell Carcinoma of Tongue. Clin Cancer Res, 14, 2588-92.

Zhang Y, Yang WQ, Zhu H, et al (2014). Regulation of autophagy by miR-30d impacts sensitivity of anaplastic thyroid carcinoma to cisplatin. Biochem Pharmacol, 87, 562-70.

Zhang Z, Liu ZB, Ren WM, et al (2012). The miR-200 family regulates the epithelial-mesenchymal transition induced by EGF/EGFR in anaplastic thyroid cancer cells. Int $\mathrm{J} \mathrm{Mol}$ Med, 30, 856-62.

Zhou H, Huang S (2011). Role of mTOR signaling in tumor cell motility, invasion and metastasis. Curr Protein Pept Sci, 12, 30-42. 